\title{
The Effect of Infinitesimal Damping on the Dynamic Instability Mechanism of Conservative Systems
}

\author{
Dimitris S. Sophianopoulos, ${ }^{1}$ George T. Michaltsos, ${ }^{2}$ and Anthony N. Kounadis ${ }^{3}$ \\ ${ }^{1}$ Department of Civil Engineering, University of Thessaly, Pedion Areos, 38334 Volos, Greece \\ ${ }^{2}$ Laboratory of Metal Structures, Department of Civil Engineering, \\ National Technical University of Athens, Zografou Campus, 15780 Athens, Greece \\ ${ }^{3}$ Research Center Pure and Applied Mathematics, Academy of Athens, Soranou Efessiou 4, \\ 11527 Athens, Greece
}

Correspondence should be addressed to Anthony N. Kounadis, kounadis@bioacademy.gr

Received 17 December 2007; Accepted 19 February 2008

Recommended by Jose Balthazar

The local instability of 2 degrees of freedom (DOF) weakly damped systems is thoroughly discussed using the Liénard-Chipart stability criterion. The individual and coupling effect of mass and stiffness distribution on the dynamic asymptotic stability due to mainly infinitesimal damping is examined. These systems may be as follows: (a) unloaded (free motion) and (b) subjected to a suddenly applied load of constant magnitude and direction with infinite duration (forced motion). The aforementioned parameters combined with the algebraic structure of the damping matrix (being either positive semidefinite or indefinite) may have under certain conditions a tremendous effect on the Jacobian eigenvalues and then on the local stability of these autonomous systems. It was found that such systems when unloaded may exhibit periodic motions or a divergent motion, while when subjected to the above step load may experience either a degenerate Hopf bifurcation or periodic attractors due to a generic Hopf bifurcation. Conditions for the existence of purely imaginary eigenvalues leading to global asymptotic stability are fully assessed. The validity of the theoretical findings presented herein is verified via a nonlinear dynamic analysis.

Copyright (C) 2008 Dimitris S. Sophianopoulos et al. This is an open access article distributed under the Creative Commons Attribution License, which permits unrestricted use, distribution, and reproduction in any medium, provided the original work is properly cited.

\section{Introduction}

In previous studies of the 3rd author, based on 2-DOF and 3-DOF cantilevered models [1] under partial follower loading (nonconservative systems), it was shown that in a small region of divergence instability, flutter (dynamic instability) may occur before divergence (static instability), if very small damping is included [2,3]. Bolotin et al. [4] using an aeroelastic model presented a similar result. Païdoussis et al. [5] and Païdoussis [6] have shown that flutter may 
occur in an inherently conservative system but for large damping. However, the effect of damping, being of paramount importance in nonconservative autonomous systems, was in general ignored when these systems are subjected to a step conservative (potential) loading. This is so because it was widely accepted that dynamic stability in nondissipative conservative systems, which are stable, does not change by the inclusion of damping [7].

The local dynamic stability of discrete systems under step conservative loading when small dissipative forces are included is governed by the matrix-vector differential equation [8-11]:

$$
\mathbf{M} \ddot{q}+\mathbf{C} \dot{q}+\mathbf{V q}=0,
$$

where the dot denotes a derivative with respect to time $t ; q(t)$ is an $n$-dimensional state vector with coordinates $q_{i}(t)(i=1, \ldots, n) ; \mathbf{M}, \mathbf{C}$ and $\mathbf{V}$ are $n \times n$ real symmetric matrices. More specifically, matrix $\mathbf{M}$ associated with the total kinetic energy of the system is a function of the concentrated masses $m_{i}(i=1, \ldots, n)$, being always positive definite; matrix $\mathbf{C}$ the elements of which are the damping coefficients $c_{i j}(i, j=1, \ldots, n)$ may be positive definite, positive semidefinite as in the case of pervasive damping $[12,13]$, or indefinite [14-16]; $\mathbf{V}$ is a generalized stiffness matrix with coefficients $k_{i j}(i, j=1, \ldots, n)$ whose elements $V_{i j}$ are also linear functions of a suddenly applied external load $\lambda$ with constant direction and infinite duration [17], that is, $V_{i j}=V_{i j}\left(\lambda ; k_{i j}\right)$. Apparently, due to this type of loading, the system under discussion is autonomous. When the external loading $\lambda$ is applied statically, one can obtain the static (divergence) instability or buckling loads $\lambda_{j}^{c}(j=1, \ldots, n)$ by vanishing of the determinant of the stiffness matrix $\mathbf{V}\left(\lambda ; k_{i j}\right)$, that is,

$$
\left|\mathbf{V}\left(\lambda ; k_{i j}\right)\right|=0
$$

Clearly, (1.2) yields an $n$th degree algebraic equation in $\lambda$. Assuming distinct critical states, the matrix $\mathbf{V}\left(\lambda ; k_{i j}\right)$ is positive definite for $\lambda<\lambda_{1}^{c}$, positive semidefinite for $\lambda=\lambda_{1}^{c}$, and indefinite for $\lambda>\lambda_{1}^{c}$.

Kounadis in two very recent publications $[10,11]$ has established the conditions under which the above autonomous dissipative systems under step (conservative) loading may exhibit dynamic bifurcational modes of instability before divergence (static) instability, that is, for $\lambda<\lambda_{1}^{c}$, when infinitesimal damping is included. These bifurcational modes may occur through either a degenerate Hopf bifurcation (leading to periodic motion around centers) or a generic Hopf bifurcation (leading to periodic attractors or to flutter). These unexpected findings (implying failure of Ziegler's kinetic criterion and other singularity phenomena) may occur for a certain combination of values of the mass (primarily) and stiffness distribution of the system in connection with a positive semidefinite or an indefinite damping matrix.

The question which now arises is whether there are combinations of values of the abovementioned parameters (mass and stiffness distribution) which in connection with the algebraic structure of damping matrices may lead to dynamic bifurcational modes of instability when the system under discussion is unloaded. Such local (due to unforced motion) dynamic instability will be sought through the set of asymptotic stability criteria of Liénard-Chipart $[8,18]$ which are elegant and more readily employed than the well-known Routh-Hurwitz stability criteria.

As another main objective of this work, some new dynamic bifurcations related to the algebraic structure of the damping matrix when the systems are loaded by the above type of step conservative load will be also discussed, using the Liénard-Chipart criterion by analyzing 
2-DOF models for which a lot of numerical results are available. Finally, the conditions of a double purely imaginary root leading to a new dynamic bifurcation, whose response is similar to that of a generic Hopf bifurcation, are properly established.

\section{Basic equations}

Solution of (1.1) can be sought in the form

$$
\mathbf{q}=\mathbf{r} e^{\rho t}
$$

where $\rho$ is in general a complex number and $\mathbf{r}$ is a complex vector independent of time $t$.

Introducing $\mathbf{q}$ from (2.1) into (1.1), we get

$$
\left(\rho^{2} \mathbf{M}+\rho \mathbf{C}+\mathbf{V}\right) \mathbf{r}=0 .
$$

For given stiffness coefficients $k_{i j}(i, j=1, \ldots, n)$, the generalized stiffness matrix $\mathbf{V}$ is a linear function of $\lambda$. Thus, if matrices $\mathbf{M}, \mathbf{C}, \mathbf{V}$ are given, solutions of (2.2) are intimately related to the algebraic properties of the matrix-valued function $\mathbf{L}(\rho)=\rho^{2} \mathbf{M}+\rho \mathbf{C}+\mathbf{V}$, and more specifically to the Jacobian eigenvalues $[\rho=\rho(\lambda)]$ obtained through the vanishing of the determinant:

$$
\left|\rho^{2} \mathbf{M}+\rho \mathbf{C}+\mathbf{V}\right|=0
$$

whose expansion gives the characteristic (secular) equation for an N-DOF system:

$$
\rho^{2 n}+\alpha_{1} \rho^{2 n-1}+\cdots+\alpha_{2 n-1} \rho+\alpha_{2 n}=0
$$

where the real coefficients $\alpha_{i}(i=1, \ldots, 2 n)$ are determined by means of Bôcher formula [19]. The eigenvalues (roots) of $(2.4) \rho_{j}(j=1, \ldots, 2 n)$ are, in general, complex conjugate pairs $\rho_{j}=$ $v_{j} \pm \mu_{j} i$ (where $v_{j}$ and $\mu_{j}$ are real numbers and $i=\sqrt{-1}$ ) with corresponding complex conjugate eigenvectors $\mathbf{r}_{j}$ and $\overline{\boldsymbol{r}}_{j}(j=1, \ldots, n)$. Since $\rho_{j}=\rho_{j}(\lambda)$, clearly $v_{j}=v_{j}(\lambda), \mu_{j}=\mu_{j}(\lambda), \mathbf{r}_{j}=\mathbf{r}_{j}(\lambda)$, and $\overline{\boldsymbol{r}}_{j}=\overline{\boldsymbol{r}}_{j}(\lambda)$. Thus, the solutions of (1.1) are of the form

$$
A e^{v_{j} t} \cos \mu_{j} t, \quad B e^{v_{j} t} \sin \mu_{j} t,
$$

where $A$ and $B$ are constants which are determined from the initial conditions. Solutions in (2.5) are bounded, tending to zero as $t \rightarrow \infty$, if all eigenvalues of (2.4) have negative real parts, that is, when $v_{j}<0$ for all $j$. In this case, the algebraic polynomial (2.4) is called a Hurwitz polynomial (since all its roots have negative real parts) and the origin $(\mathbf{q}=\dot{\mathbf{q}}=0)$ of the system is asymptotically stable.

\subsection{Criteria for asymptotic stability}

The necessary and sufficient conditions which assure that all roots of (2.4) have negative real parts (i.e., $v_{j}<0$ for all $j$ ) which means that the corresponding polynomial $|L(\rho)|$ is a Hurwitz polynomial are of great practical importance. 
Consider the more general case of a polynomial in $z$ with real coefficients $\alpha_{i}(i=1, \ldots, n)$ :

$$
f(z)=\alpha_{0} z^{n}+\alpha_{1} z^{n-1}+\cdots+\alpha_{n-1} z+\alpha_{n}=0 \quad\left(\alpha_{0}>0\right)
$$

for which we will seek the necessary and sufficient conditions so that all its roots have negative real parts.

Denoting by $z_{\mathcal{K}}(\kappa=1, \ldots, m)$ the real roots and by $\left.r_{j} \pm i s_{j}(j=1, \ldots,(n-m) / 2) ; i=\sqrt{-1}\right)$ the complex roots of (2.6), we may assure that all these roots in the complex plane lie to the left of the imaginary axis, that is,

$$
z_{\kappa}<0, \quad r_{j}<0 \quad\left(\kappa=1, \ldots, m ; j=1, \ldots, \frac{n-m}{2}\right)
$$

Then one can write

$$
f(z)=\alpha_{0} \prod_{\kappa=1}^{m}\left(z-z_{\kappa}\right) \prod_{j=1}^{n-m}\left(z^{2}-2 r_{j} z+r_{j}^{2}+s_{j}^{2}\right) .
$$

Since due to inequality (2.7), each term in the last part of (2.8) has positive coefficients, it is deduced that all coefficients of (2.6) are also positive. However, this (i.e., $\alpha_{i}>0$ for all $i$ with $\alpha_{0}>0$ ) is a necessary but by no means sufficient condition for all roots of (2.6) to lie in the left half-plane (i.e., $\operatorname{Re}(z)<0$ ).

According to Routh-Hurwitz criterion [18] of asymptotic stability for all roots of (2.6) to have negative real parts, the necessary and sufficient conditions are

$$
\Delta_{1}>0, \Delta_{2}>0, \ldots, \Delta_{n}>0
$$

where

$$
\Delta_{1}=\alpha_{1}, \Delta_{2}=\left|\begin{array}{cc}
\alpha_{1} & \alpha_{3} \\
\alpha_{0} & \alpha_{2}
\end{array}\right|, \Delta_{3}=\left|\begin{array}{ccc}
\alpha_{1} & \alpha_{3} & 0 \\
\alpha_{0} & \alpha_{2} & \alpha_{4} \\
0 & \alpha_{1} & \alpha_{3}
\end{array}\right|, \ldots, \Delta_{\mathrm{n}}=\left|\begin{array}{ccccccc}
\alpha_{1} & \alpha_{3} & \alpha_{5} & \ldots & \ldots & \ldots & \ldots \\
\alpha_{0} & \alpha_{2} & \alpha_{4} & \ldots & \ldots & \ldots & \ldots \\
0 & \alpha_{1} & \alpha_{3} & \ldots & \ldots & \ldots & \ldots \\
0 & \alpha_{0} & \alpha_{2} & \alpha_{4} & \ldots & \ldots & \ldots \\
\ldots & \ldots & \ldots & \ldots & \ldots & \ldots & \ldots \\
\ldots & \ldots & \ldots & \ldots & \ldots & \ldots & \ldots \\
\ldots & \ldots & \ldots & \ldots & \ldots & \ldots & \alpha_{i}
\end{array}\right|
$$

(with $\alpha_{\kappa}=0$ for $\kappa>n$ ). Note the last equality $\Delta_{n}=\alpha_{n} \Delta_{n-1}$.

It should be noted that when the above necessary conditions $\alpha_{i}>0$ (for all $i$ ) hold, inequalities (2.9) are not independent. For instance, for $n=4$, the Routh-Hurwitz conditions reduce to the single inequality $\Delta_{3}>0$; for $n=5$, they reduce to $\Delta_{2}>0$ and $\Delta_{4}>0$; while for $n=6$, they reduce also to two inequalities, $\Delta_{3}>0, \Delta_{5}>0$. This case was discussed by Liénard and Chipart who established the following elegant criterion for asymptotic stability [8]. 


\section{The Liénard-Chipart stability criterion}

For a polynomial with real coefficients $f(z)=\alpha_{0} z^{n}+\alpha_{1} z^{n-1}+\cdots+\alpha_{n-1} z+\alpha_{n}=0\left(\alpha_{0}>0\right)$ to have all roots with negative real parts, it is necessary and sufficient that

(1) all coefficients of $f(z)$ be positive, that is,

$$
\alpha_{i}>0 \quad(i=1, \ldots, n)
$$

(2) the determinant inequalities be also positive, that is,

$$
\Delta_{n-1}>0, \Delta_{n-3}>0, \ldots,
$$

where $\Delta_{\kappa}$ denotes as before the Hurwitz determinant of $\kappa$ th order.

It can be shown that if the Hurwitz determinants of odd order are positive, then those of even order are also positive, and vice versa. This holds even when only part of the coefficients $\alpha_{i}$ of $f(z)$ (with $\alpha_{0}>0$ ) are positive. According to this, the Lienard-Chipart criterion is defined as follows.

Necessary and sufficient conditions for all roots of the real polynomial $f(z)=\alpha_{0} z^{n}+$ $\alpha_{1} z^{n-1}+\cdots+\alpha_{n-1} z+\alpha_{n}=0,\left(\alpha_{0}>0\right)$ to have negative real parts can be given in any one of the following forms [18]:

(1)

$$
\alpha_{n}>0, \alpha_{n-2}>0, \ldots ; \text { with } \begin{cases}\text { either } \Delta_{1}>0, & \Delta_{3}>0, \ldots, \\ \text { or } \Delta_{2}>0, & \Delta_{4}>0, \ldots,\end{cases}
$$

(2)

$$
\alpha_{n}>0, \alpha_{n-1}>0, \alpha_{n-3}>0, \ldots ; \text { with } \begin{cases}\text { either } \Delta_{1}>0, & \Delta_{3}>0, \ldots \\ \text { or } \Delta_{2}>0, & \Delta_{4}>0, \ldots\end{cases}
$$

This stability criterion was rediscovered by Fuller [20].

For instance, for a 2-DOF cantilevered model, the characteristic (secular) (2.4) is

$$
\rho^{4}+\alpha_{1} \rho^{3}+\alpha_{2} \rho^{2}+\alpha_{3} \rho+\alpha_{4}=0 \quad\left(\alpha_{0}=1\right)
$$

According to the last criterion, all roots of (2.13) have negative real parts provided that $\alpha_{4}>0, \alpha_{2}>0, \Delta_{1}=\alpha_{1}>0$ and $\Delta_{3}=\alpha_{3}\left(\alpha_{1} \alpha_{2}-\alpha_{3}\right)-\alpha_{1}^{2} \alpha_{4}>0$. Clearly, from the last inequality, it follows that $\alpha_{3}>0$. Hence, the positivity of $\alpha_{1}$ and $\alpha_{3}$ was assured via the above conditions (i.e., $\alpha_{4}>0, \alpha_{2}>0, \Delta_{1}>0, \Delta_{3}>0$ ). 


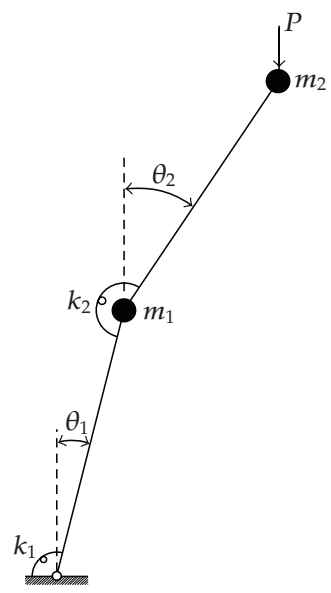

Figure 1: 2-DOF autonomous system under conservative loading.

\section{Mathematical analysis}

Subsequently, using the spring cantilevered dynamical model of 2-DOF shown in Figure 1, we will examine in detail the effect of violation of one or more of the conditions of LiénardChipart criterion on its asymptotic stability. The response of this dynamic model carrying two concentrated masses is studied when it is either unloaded or loaded by a suddenly applied load of constant magnitude and direction with infinite duration. Such autonomous dissipative systems with infinitesimal damping (including the case of zero loading) are properly discussed. If at least one root of the secular equation (2.13) has a positive real part, the corresponding solution (2.5) will contain an exponentially increasing function and the system will become unstable.

The seeking of an imaginary root of the secular equation (2.13) which represents a border line between dynamic stability and instability is a first but important step in our discussion. Clearly, an imaginary root gives rise to an oscillatory motion of the form $e^{i \mu t},(i=$ $\sqrt{-1}, \mu$ real number) around the trivial state. However, the existence of at least one multiple imaginary root of the $\kappa$ th order of multiplicity leads to a solution containing functions of the form $e^{i \mu t}, t e^{i \mu t}, \ldots, t^{\kappa-1} e^{i \mu t}$ which increases with time. Hence, the multiple imaginary root on the imaginary axis of the complex plane denotes local dynamic instability. The discussion of such a situation is also another objective of this study.

The nonlinear equations of motion for the 2-DOF model of Figure 1 with rigid links of equal length $\ell$ are given by [11]

$$
\begin{gathered}
(1+m) \ddot{\theta}_{1}+\ddot{\theta}_{2} \cos \left(\theta_{1}-\theta_{2}\right)+\dot{\theta}_{2}^{2} \sin \left(\theta_{1}-\theta_{2}\right)+c_{11} \dot{\theta}_{1}+c_{12} \dot{\theta}_{2}+k \theta_{1}-\theta_{2}+\theta_{1}-\lambda \sin \theta_{1}=0, \\
\ddot{\theta}_{2}+\ddot{\theta}_{1} \cos \left(\theta_{1}-\theta_{2}\right)-\dot{\theta}_{1}^{2} \sin \left(\theta_{1}-\theta_{2}\right)+c_{22} \dot{\theta}_{2}+c_{12} \dot{\theta}_{1}-\theta_{1}+\theta_{2}-\lambda \sin \theta_{2}=0,
\end{gathered}
$$

where $m=m_{1} / m_{2}, k=k_{1} / k_{2}, \lambda=P \ell / k_{2}$.

Linearization of (3.1) after setting

$$
\Theta=\left[\begin{array}{l}
\theta_{1} \\
\theta_{2}
\end{array}\right]=e^{\rho t}\left[\begin{array}{l}
\varphi_{1} \\
\varphi_{2}
\end{array}\right]=e^{\rho t} \boldsymbol{\varphi}
$$


gives

$$
\left(\rho^{2} \mathbf{M}+\rho \mathbf{C}+\mathbf{V}\right) \boldsymbol{\varphi}=0
$$

where

$$
\mathbf{M}=\left[\begin{array}{ll}
m_{11} & m_{12} \\
m_{12} & m_{22}
\end{array}\right]=\left[\begin{array}{cc}
1+m & 1 \\
1 & 1
\end{array}\right], \quad \mathbf{C}=\left[\begin{array}{ll}
c_{11} & c_{12} \\
c_{12} & c_{22}
\end{array}\right], \quad \mathbf{V}=\left[\begin{array}{ll}
V_{11} & V_{12} \\
V_{12} & V_{22}
\end{array}\right]=\left[\begin{array}{cc}
k+1-\lambda & -1 \\
-1 & 1-\lambda
\end{array}\right] .
$$

Note that in case of a Rayleigh's dissipative function the damping coefficients are, $c_{11}=$ $c_{1}+c_{2}, c_{12}=-c_{2}, c_{21}=-c_{2}$, and $c_{22}=c_{2}$, where $c_{i}(i=1,2)$ is dimensionless coefficient for the $i$ th rigid link. This case (for which $\operatorname{det} \mathbf{C}=|c|=c_{1} c_{2}$ ) is a specific situation of the damping matrix $\mathrm{C}$ which is not discussed herein.

The static buckling (divergence) equation is given by

$$
\lambda^{2}-(2+k) \lambda+k=0,
$$

whose lowest root is the first buckling load $\lambda_{1}^{c}$ equal to

$$
\lambda_{1}^{c}=0.5\left(k+2-\sqrt{k^{2}+4}\right) .
$$

Clearly, for the entire interval of values of $k>0$, (3.6) yields $0 \leq \lambda_{1}^{c}<1$.

The characteristic (secular) equation is

$$
\rho^{4}+\alpha_{1} \rho^{3}+\alpha_{2} \rho^{2}+\alpha_{3} \rho+\alpha_{4}=0
$$

where

$$
\begin{aligned}
& \alpha_{1}=\frac{1}{m}\left(m_{11} c_{22}+m_{22} c_{11}-2 m_{12} c_{12}\right), \\
& \alpha_{2}=\frac{1}{m}\left(m_{11} V_{22}+m_{22} V_{11}-2 V_{12} m_{12}+|c|\right), \\
& \alpha_{3}=\frac{1}{m}\left(c_{11} V_{22}+c_{22} V_{11}-2 V_{12} c_{12}\right), \\
& \alpha_{4}=\frac{1}{m}\left(V_{11} V_{22}-V_{12}^{2}\right) .
\end{aligned}
$$

Let us first examine the effect of violation of Lienard-Chipartcriterion on the system stabilityin the case of zero loading (i.e., $\lambda=0$ ). Then expressions in (3.8) due to relations (3.4) are written as follows:

$$
\begin{aligned}
& \alpha_{1}=\frac{1}{m}\left[(1+m) c_{22}+c_{11}-2 c_{12}\right], \\
& \alpha_{2}=\frac{1}{m}\left[k+m+4+c_{11} c_{22}-c_{12}^{2}\right], \\
& \alpha_{3}=\frac{1}{m}\left[c_{11}+c_{22}(k+1)+2 c_{12}\right], \\
& \alpha_{4}=\frac{k}{m}
\end{aligned}
$$


According to Liénard-Chipart criterion, inequalities (2.12a) imply

$$
\alpha_{4}>0, \quad \alpha_{2}>0, \quad \Delta_{1}, \Delta_{3}>0,
$$

where $\Delta_{1}=\alpha_{1}>0$ and $\Delta_{3}=\alpha_{3}\left(\alpha_{1} \alpha_{2}-\alpha_{3}\right)-\alpha_{1}^{2} \alpha_{4}>0$. Clearly, from the last inequality, it follows that $\alpha_{3}>0$.

For $c_{i i}>0(i=1,2), k>0$, and $m>0$ (implying $\alpha_{4}>0$ ), it is deduced that this criterion is violated if either one of $\alpha_{1}$ or $\alpha_{2}$ is zero or $\Delta_{3}$ is zero. These three cases will be discussed separately in connection with the algebraic structure of the damping matrix $\mathbf{C}=\left[c_{i j}\right]$.

Case $1\left(\alpha_{1}=0\right.$ with $\left.\alpha_{2}>0\right)$. If $\alpha_{1}=0$ (yielding $\left.\Delta_{3}=-\alpha_{3}^{2}<0\right)$, then

$$
(1+m) c_{22}+c_{11}-2 c_{12}=0
$$

Equation (3.11), being independent of $\lambda$ and $k$, is satisfied only when the damping matrix $\mathbf{C}$ is indefinite, that is,

$$
c_{11} c_{22}-c_{12}^{2}<0 \quad\left(c_{i i}>0 \text { for } i, j=1,2\right) .
$$

Indeed, the last inequality due to relation (3.11) implies

$$
(1+m)^{2} c_{22}^{2}+2(m-1) c_{11} c_{22}+c_{11}^{2}>0
$$

which is always satisfied, regardless of the value of $c_{22} / c_{11}$, since for $m>0$, the discriminant of (3.13) (equal to $-16 c_{11}^{2} m$ ) is always negative.

Thus, we have explored the unexpected finding that an unloaded (stable) system becomes dynamically unstable at any small disturbance in case of an indefinite damping matrix even when infinitesimal damping is included.

Since all coefficients of (3.7) are positive from the theory of algebraic equations it follows that this equation cannot have positive root. Also the case of existence of a pair of pure imaginary roots associated with $\Delta_{3}=0$ is ruled out, since $\Delta_{3}<0$ (due to $\alpha_{1}=0$ ). Hence, (3.7) has either two negative roots combined with a pair of complex conjugate roots with positive real part or two pairs of complex conjugate roots with opposite real parts. Both cases imply local dynamic instability.

Case $2\left(\alpha_{2}=0\right.$ with $\left.\alpha_{1}>0\right)$. If $\alpha_{2}=0$ (implying also $\left.\Delta_{3}<0\right)$, then

$$
|c|=-k-m-4<0 \quad(k, m>0) .
$$

Namely, the damping matrix $\left[c_{i j}\right]$ is indefinite but with large negative determinant (rather unrealistic case). Since the Liénard-Chipart criterion is violated, the model is again locally dynamically unstable.

Since all coefficients of (3.7) are positive, from the theory of algebraic equations, it is deduced that this equation cannot have positive root. Also the case of existence of a pair of pure imaginary roots associated with $\Delta_{3}=0$ is ruled out, since $\Delta_{3}<0$ (due to $\alpha_{2}=0$ ). Hence, (3.7) has either two negative roots combined with a pair of complex conjugate roots with positive real part or two pairs of complex conjugate roots with opposite real parts. Both cases imply local dynamic instability. 
Case $3\left(\Delta_{3}=0\right)$. In this case, stability conditions in (3.10) are satisfied except for the last one, since $\Delta_{3}=0$ which yields [11]

$$
\Delta_{3}=\alpha_{3}\left(\alpha_{1} \alpha_{2}-\alpha_{3}\right)-\alpha_{1}^{2} \alpha_{4}=0 .
$$

Note that $\lambda<\lambda_{1}^{c}$ implies $\alpha_{4}>0$ (i.e., $\operatorname{det} \mathbf{V}>0$ ).

This is a necessary condition for the secular (3.7) to have one pair of pure imaginary roots $\pm \mu i,(i=\sqrt{-1})$. Indeed, this can be readily established by inserting $\rho= \pm \mu i$ into (3.7) and then equating to zero real and imaginary parts.

Consider now the more general case of nonzero loading (i.e., $\lambda \neq 0$ ). Using relations in (3.8), (3.15) can be written as follows:

$$
A \curlywedge^{2}+B \curlywedge+\Gamma=0
$$

where

$$
\begin{aligned}
A= & \left(c_{11}+c_{22}\right)(m+2) \bar{\alpha}_{1}-m\left(c_{11}+c_{22}\right)^{2}-\left[(1+m) c_{22}+c_{11}-2 c_{12}\right]^{2}, \\
B= & -\bar{\alpha}_{1}\left\{(m+2)\left[c_{11}+(k+1) c_{22}+2 c_{12}\right]+\left(c_{11}+c_{22}\right)[k+m+4+|c|]\right\} \\
& +2 m\left[c_{11}+(k+1) c_{22}+2 c_{12}\right]\left(c_{11}+c_{22}\right)+(k+2)\left[(1+m) c_{22}+c_{11}-2 c_{12}\right]^{2}, \\
\Gamma= & (k+m+4+|c|)\left[c_{11}+(k+1) c_{22}+2 c_{12}\right] \bar{\alpha}_{1}-m\left[c_{11}+(k+1) c_{22}+2 c_{12}\right]^{2} \\
& -k\left[(1+m) c_{22}+c_{11}-2 c_{12}\right]^{2},
\end{aligned}
$$

where

$$
c_{11}=\frac{c_{12}^{2}+|c|}{c_{22}}, \quad \bar{\alpha}_{1}=m \alpha_{1}
$$

For real $\lambda$, the discriminant $\mathbf{D}$ of (3.16) must be greater than or equal to zero, that is,

$$
\mathbf{D}=B^{2}-4 A \Gamma \geq 0 .
$$

Subsequently, attention is focused on the following: (a) matrix $\mathrm{C}$ is positive semidefinite (i.e., $|c|=0$ with $\left.c_{i i}>0, i=1,2\right)$ and (b) matrix $\mathbf{C}$ is indefinite $\left(|c|<0\right.$ with $\left.c_{i i}>0, i=1,2\right)$.

Using the symbolic manipulation of Mathematica [21], one can find that

$$
\mathbf{D}=|c| f(|c|),
$$

where $f(|c|)$ is an algebraic polynomial of 5 th degree in $|c|$.

Case $4(|c|=0, f \neq 0)$. For $|c|=0,(3.16)$ implying $\mathbf{D}=0$ admits a double root, which due to (3.17a), (3.17b), (3.17d) is given by

$$
\lambda_{1}^{H}=\frac{2 c_{12}^{2}+c_{12} c_{22}(k-m)-c_{22}^{2}(k+m+2)}{c_{12}^{2}-c_{22}^{2}-c_{12} c_{22} m} .
$$

Using the Reduce command embedded in Mathematica, one can find the conditions under which $0<\lambda_{1}^{H}<\lambda_{1}^{c}$, given in the appendix, relation (A.1). 
Case $5(f=0,|c| \neq 0)$. Moreover, it was found symbolically that the 5 th degree polynomial $f(|c|)$ possesses three real roots (one double and one single), and two pure imaginary ones. Discussing their nature, one can find that the double root of $f(|c|)$, being equal to $|c|=-\left(c_{12}-c_{22}\right)^{2}-c_{22}^{2} m<0$, yields

$$
c_{12}=\frac{1}{2}\left[c_{11}+(m+1) c_{22}\right]
$$

Then, the double root of (3.16) becomes

$$
\lambda_{2}^{H}=\frac{2 c_{11}+c_{22}(k+m+2)}{c_{11}+c_{22}}
$$

which is always greater than $\lambda_{1}^{c}$ and hence of minor importance for the present analysis.

The third real root of $f(|c|)$, if substituted in (3.16), yields again a double root in $\lambda$, always less than zero, which is rejected. Thus, only the case of a positive semidefinite damping matrix may lead to an acceptable value of the corresponding load (i.e., $0<\lambda_{H}<\lambda_{1}^{c}$ ) associated with a degenerate Hopf bifurcation, as theoretically was shown by Kounadis [10, 11].

Case $6(\lambda=0)$. If $\lambda=0$, (3.16) implies $\Gamma=0$, which after symbolic manipulation of (3.17c) can be written in the following form:

$$
\Gamma=\frac{1}{c_{22}^{2}}\left(|c|^{3}+A_{2}|c|^{2}+A_{1}|c|+A_{0}\right)=0,
$$

where $A_{i}(i=0,1,2)$ are given in the appendix, relations (A.2). It is evident that $A_{2}>0$ and $A_{0} \geq 0$, a fact implying that (3.23) can be satisfied only for $|c| \leq 0$ if also $A_{1} \geq 0$; otherwise (i.e., if $\left.A_{1}<0\right)$ the system may be locally stable or unstable. For $|c|=0$, one can find the corresponding values of $c_{i i}(i=1,2)$, given in (A.3) and (A.4) in the appendix, which are always positive. This special case, for which the trivial (unloaded) state is associated with a pair of pure imaginary eigenvalues (necessary condition for a Hopf bifurcation), implies a local dynamic instability.

\section{Conditions for a double imaginary root}

For a double imaginary root, the first derivative of the secular equation (3.7) must be also zero, which yields

$$
4 \rho^{3}+3 \alpha_{1} \rho^{2}+2 \alpha_{2} \rho+\alpha_{3}=0 .
$$

Inserting into (3.24) $\rho=\mu i$, we obtain $\mu^{2}=0.5 \alpha_{2}=\alpha_{3} / 3 \alpha_{1}$ and thus $\alpha_{3}=3 \alpha_{1} \alpha_{2} / 2$. Introducing this expression of $\alpha_{3}$ into (3.15), it follows that $\alpha_{1}=0$, which also implies that $\alpha_{3}=0$ and hence (3.24) becomes $\rho^{2}=-0.5 \alpha_{2}$. If $\rho^{2}=-0.5 \alpha_{2}$ is inserted into the secular equation $\rho^{4}+\alpha_{2} \rho^{2}+\alpha_{4}=0$, for a double imaginary root, it follows that $\alpha_{2}^{2}-4 \alpha_{4}=0$ which due to relations (3.8) yields

$$
\begin{aligned}
\left(m^{2}+4\right) \lambda^{2} & -2 \lambda\left(m^{2}+(2-k) m+2 k+8+(m+2)|c|\right) \mid \\
+ & |c|^{2}+2(k+m+4)|c|+(k+m+4)^{2}-4 m k=0 .
\end{aligned}
$$


For real $\lambda$, the discriminant $\mathbf{D}$ of (3.25) must satisfy the inequality

$$
\mathbf{D}=|c|^{2}+(4-m k)|c|-(m+k)^{2} \geq 0
$$

which yields

$$
\begin{gathered}
\text { either }|c|<0.5\left(m k-4-\sqrt{m^{2}\left(k^{2}+4\right)+4 k^{2}+16}\right), \\
\text { or }|c|>0.5\left(m k-4+\sqrt{m^{2}\left(k^{2}+4\right)+4 k^{2}+16}\right) .
\end{gathered}
$$

Using the conditions found above

$$
\alpha_{1}=\alpha_{3}=0,
$$

relations (3.9) yield

$$
\begin{gathered}
(1+m) c_{22}+c_{11}-2 c_{12}=0, \\
c_{11}(1-\lambda)+c_{22}(k+1-\lambda)+2 c_{12}=0 .
\end{gathered}
$$

Adding the last two equations, we obtain

$$
(2-\lambda) c_{11}+(k+m+2-\lambda) c_{22}=0 .
$$

Since $k, m>0$, and $\lambda<\lambda_{1}^{c}<1$, it follows that both coefficients of $c_{11}$ and $c_{22}$ in (3.30) are positive. Hence, $c_{11}$ and $c_{22}$ are of opposite sign (i.e., $c_{11} / c_{22}<0$ ) and consequently $|c|=c_{11} c_{22}-c_{12}^{2}<0$; thus the 2 nd of inequalities (3.27) is excluded.

Solving simultaneously the system of equations $\alpha_{2}^{2}-4 \alpha_{4}=0, \alpha_{1}=\alpha_{3}=0$ in $k, m, \lambda$, two ternaries of values for $k, m$, and $\lambda$ are obtained, given in the appendix, expressions (A.5). For all these values to be greater than zero, the Reduce command embedded in Mathematica [21] yields two sets of conditions, given also in the appendix, relations (A.6). Further symbolic computations are needed for establishing the conditions for a double pure imaginary root for loading values less than $\lambda_{1}^{c}$. Nevertheless, suitable combinations of values of $c_{i j}, k$, and $m$ may be found. This will be demonstrated in Section 4 . The corresponding dynamic response, since the system is associated with a codimension-2 local bifurcation, is anticipated to be related to isolated periodic orbits which will be established via a straightforward complete nonlinear dynamic analysis.

\section{Numerical results}

In this section, numerical results corresponding to all the above cases of violation of the Liénard-Chipart stability criterion are given below in tabular and graphical forms.

Case $1\left(\alpha_{1}=0\right)$. (a) $\lambda=0$. For an unloaded system with $k=m=1$, choosing $c_{11}=0.015$ and $c_{22}=0.002$, (3.11) yields $c_{12}=0.0095$ and as expected the damping matrix is indefinite with determinant $|c|=-0.0006025$. The two pairs of corresponding eigenvalues are $\rho_{1,2}=-0.00332577 \pm 0.41421 i$ and $\rho_{3,4}=0.00335877 \pm 0.41421 i(i=\sqrt{-1})$, implying local dynamic instability. Solving numerically the system of nonlinear equations (3.1), we find that the 
dynamic response of the system is associated with a divergent motion, as depicted in Figure 2, with the aid of the time series

$\left[\tau, \theta_{1}(\tau)\right]$, time velocities $\left[\tau, \dot{\theta}_{1}(\tau)\right]$ and phase-plane portraits $\left[\theta_{1}(\tau), \dot{\theta}_{1}(\tau)\right]$.

(b) $\lambda \neq 0$. For a system with $k=5, m=4$, and $\lambda=0.5<\lambda_{1}^{c}=0.807418$ and for $c_{11}=$ $0.01, c_{22}=0.002$, (2.13) yields $c_{12}=-0.0175$ implying $|c|=-2.5625 \times 10^{-4}$. The trivial state is locally dynamically unstable, since $\rho_{1,2}=-0.00397748 \pm 0.4351 i$ and $\rho_{3,4}=0.00397748 \pm 0.4351 i$. The corresponding dynamic response is again related to a divergent (unbounded) motion, as shown in the phase-plane portraits of Figures 3(a) and 3(b).

Case $2\left(\alpha_{2}=0\right)$. (a) $\lambda=0$ : If $k=m=1$ relation (3.14) is satisfied, for example, for the damping coefficients $c_{11}=0.50, c_{22}=2.00$, yielding $c_{12}=\sqrt{7}$ and $|c|=-6$. For this, rather unrealistic, subcase, the corresponding eigenvalues are equal to $\rho_{1}=-1.86617, \rho_{2}=-0.102227$, and $\rho_{3,4}=1.37995 \pm 1.8269 i$ (local instability). Hence, the response of the system is also related to a divergent (unbounded) motion, presented graphically in the phase-plane curves of Figures 4(a) and $4(b)$.

(b) $\lambda \neq 0$. Similarly, for a system with $k=0.10, m=0.20$ (for which $\lambda_{1}^{c}=0.0487508$ ) in order that $\alpha_{2}=0$, we must choose an indefinite damping matrix with $|c|=-4.25$. Setting, for example, $c_{11}=2.375, c_{12}=3.00, c_{22}=2.00$, and $\lambda=0.227273<\lambda_{1}^{c}$, the trivial state is locally dynamically unstable with $\rho_{1}=-2.46657, \rho_{2}=-0.00503929$, and $\rho_{3,4}=4.2983 \pm 1.6612 i$. The system exhibits a divergent (unbounded) motion, as shown in Figures 5(a) and 5(b).

Case $3\left(\Delta_{3}=0\right.$ with $\left.\alpha_{1} \alpha_{2} \neq 0\right)$. (a) Positive semidefinite damping matrix $(|c|=0)$. Choosing $c_{11}=$ $0.01, c_{22}=0.0004$ (and thus $c_{12}=0.002$ ), the 1st requirement of the 2 nd set of conditions given in the appendix, relation (A.1), is satisfied (i.e., $c_{12}>c_{22}$ ). The 2 nd requirement, that is, $m>2\left(c_{12}-c_{22}\right) / c_{22}$, yields $m>8$, and hence one can choose $m=10$. The 3 rd requirement in (A.1) implies that $0.8333<k<3$ and thus one can take $k=1$. Solving numerically (3.15) with respect to $\lambda$, we obtain the value of $\lambda_{H}=0.307692256<\lambda_{1}^{c}=0.381966$, associated with a pair of pure imaginary eigenvalues, while the other pair has negative real parts. The evolution of both pairs of eigenvalues in the complex plane as $\lambda$ varies is presented in Figure 6 for $\lambda<\lambda_{1}^{c}$. For $\lambda=\lambda_{H}$, a degenerate Hopf bifurcation occurs and the system exhibits a periodic motion, whose amplitude depends on the initial conditions. Relevant results in graphical form can be found in recent publications of the 3rd author $[10,11]$.

(b) Indefinite damping matrix $(|c|<0)$. It has been proven by Kounadis $[10,11]$ that in this subcase (for $\lambda<\lambda_{1}^{c}$ ) all the necessary and sufficient conditions for a generic Hopf bifurcation are fulfilled and hence the system experiences a periodic attractor response (stable limit cycles) with constant final amplitudes regardless of the initial conditions. Numerical results are given in the aforementioned papers by Kounadis.

(c) $\Delta_{3}=0$ and $\lambda=0$. If at the same time $|c|=0$, one can find the values of $c_{i i}(i=1,2)$ through (A.3) and (A.4) in the appendix, which are always positive. A further investigation of this case as well as of the case $|c|<0$ for the global stability of the system can be performed through a nonlinear dynamic analysis.

(d) Double pure imaginary eigenvalues. For this special case, three combinations of damping matrix coefficients $c_{i j}$ are examined. These, along with the corresponding critical values of $k, \lambda$ and $m$, satisfying relations (A.5) of the appendix, are given in Table 1 . Note that Cases $3(d) 1$ and 3(d) 2 are the outcome of the 1st set from relations (A.5), while Case 3(d)3 is the outcome from the 2 nd set. Clearly, in all cases, $\lambda<\lambda_{1}^{c}$. 
Dimitris S. Sophianopoulos et al.

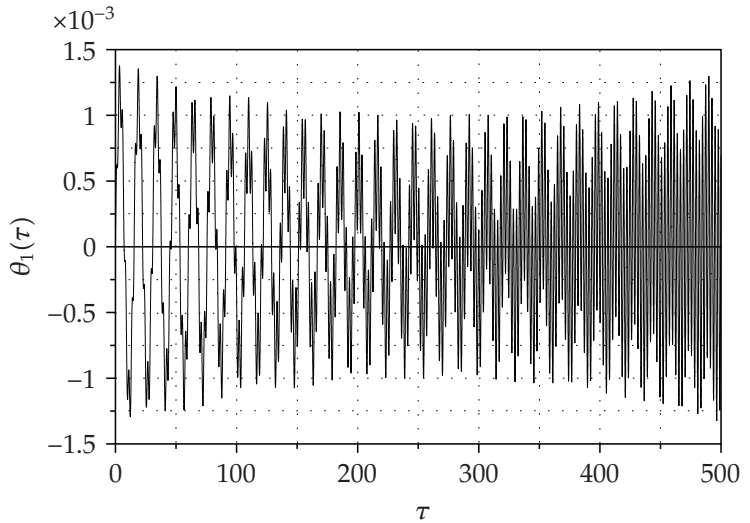

(a)

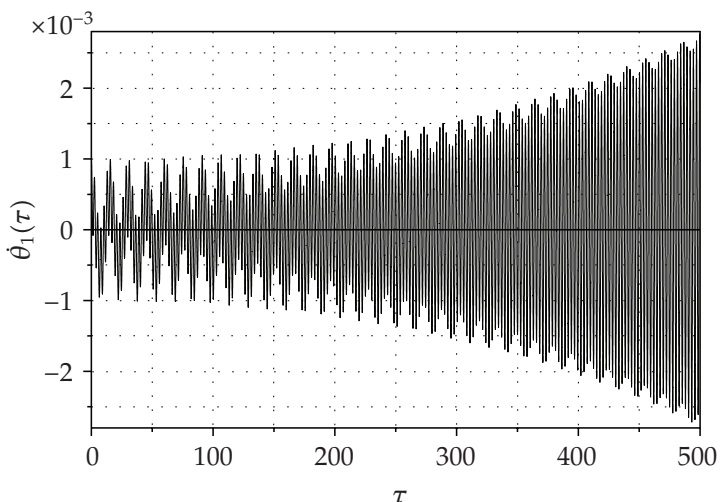

(b)

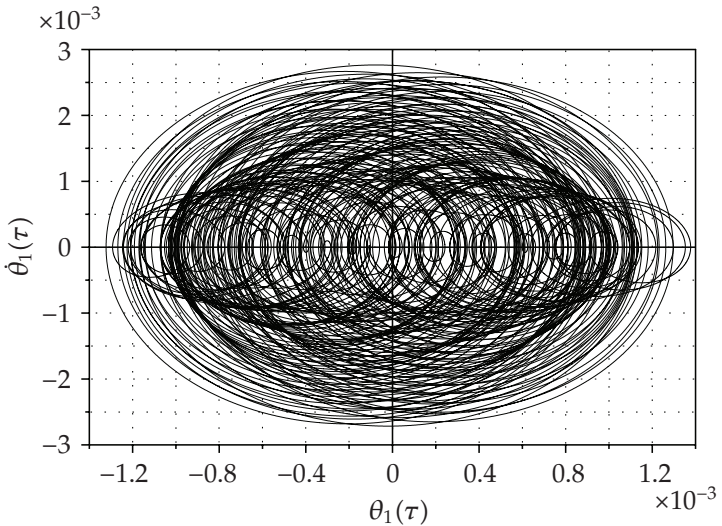

(c)

Figure 2: (a) Time series $\left[\tau, \theta_{1}(\tau)\right]$, (b) time velocities $\left[\tau, \dot{\theta}_{1}(\tau)\right]$, and (c) phase-plane portraits $\left[\theta_{1}(\tau), \dot{\theta}_{1}(\tau)\right]$, for the system of Case 1(a), exhibiting a divergent motion. 


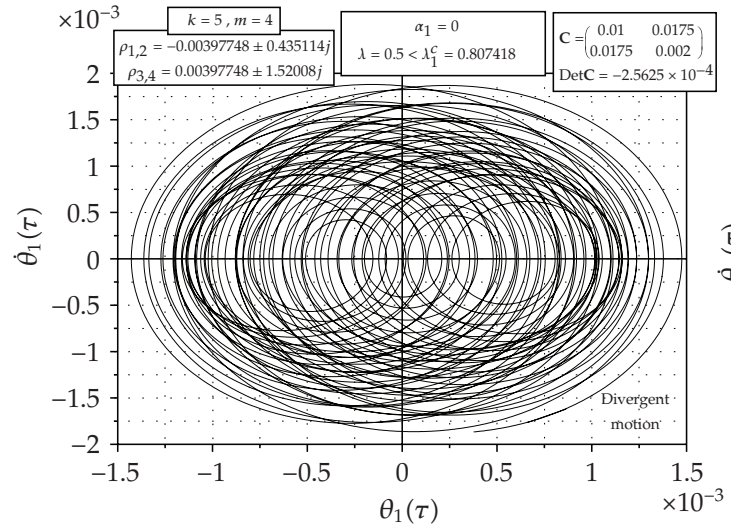

(a)

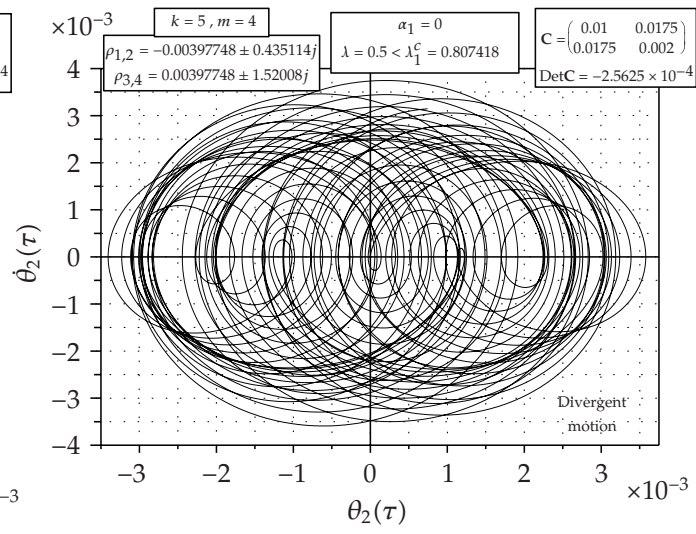

(b)

Figure 3: Phase-plane portraits $\left[\theta_{i}(\tau), \dot{\theta}_{i}(\tau)\right](i=1,2)$ for the system of Case $1(\mathrm{~b})$, associated with a divergent motion.

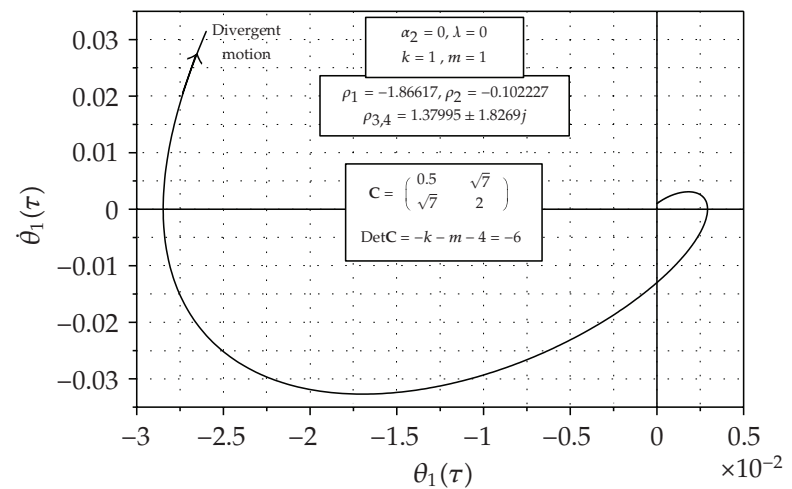

(a)

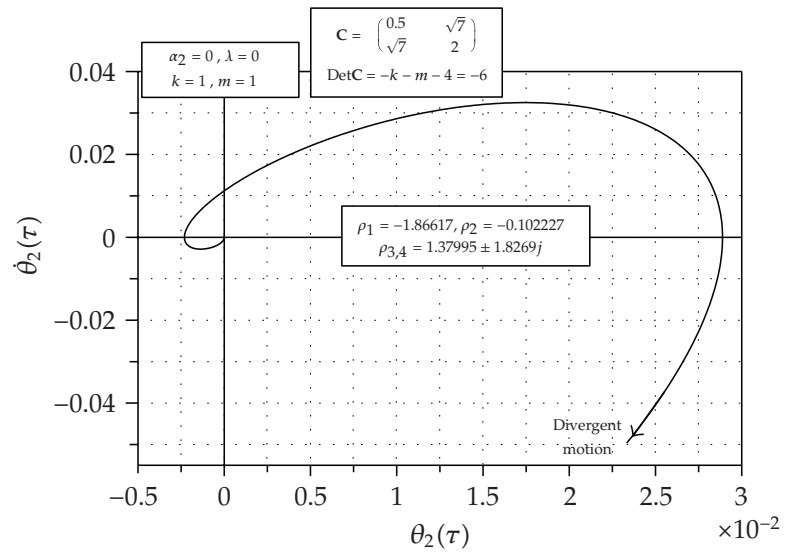

(b)

Figure 4: Phase-plane portraits $\left[\theta_{i}(\tau), \dot{\theta}_{i}(\tau)\right](i=1,2)$ for the system of Case 2(a), associated with a divergent motion. 


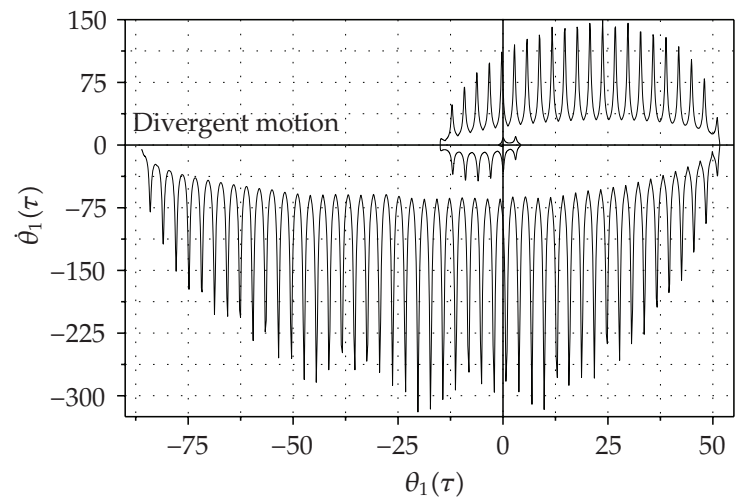

(a)

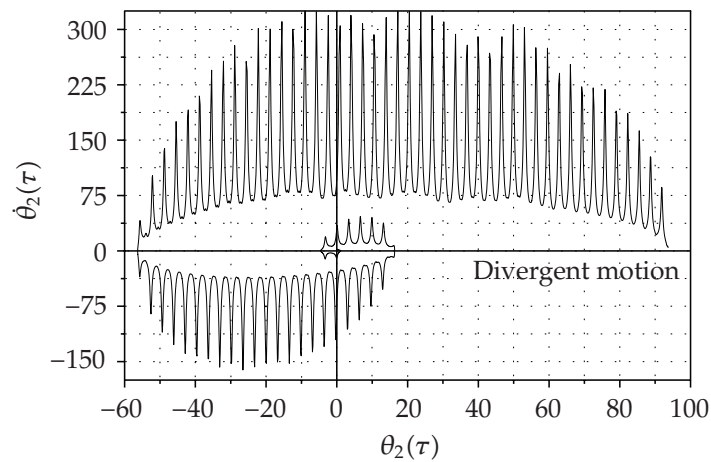

(b)

Figure 5: Phase-plane portraits $\left[\theta_{i}(\tau), \dot{\theta}_{i}(\tau)\right](i=1,2)$ for the system of Case 2(b), associated with a divergent motion.

Table 1: Values of damping coefficients $\left(c_{i j}\right)$ and critical system parameters $\left(k, \lambda_{c r}, m\right)$ for three subcases with double pure imaginary eigenvalues.

\begin{tabular}{c|ccc|cccc}
\hline Case no. & $c_{11}$ & $\begin{array}{c}c_{12} \\
|c|\end{array}$ & $c_{22}$ & $k$ & $\lambda_{\text {cr }}$ & $m$ & $\lambda_{1}^{c}$ \\
\hline $3(\mathrm{~d}) 1$ & 3.45 & $\begin{array}{c}1.00 \\
-5.83\end{array}$ & -1.40 & 2.65201108 & 0.164480292 & 0.035714286 & 0.665195 \\
\hline $3(\mathrm{~d}) 2$ & 3.60 & $\begin{array}{c}1.00 \\
-5.32\end{array}$ & -1.20 & 3.178585026 & 0.244040821 & 0.333333333 & 0.711568 \\
\hline $3(\mathrm{~d}) 3$ & 3.40 & $\begin{array}{c}1.00 \\
-5.08\end{array}$ & -1.20 & 2.346316274 & 0.629282032 & 0.166666666 & 0.631633 \\
\hline
\end{tabular}

In the three above subcases, the evolution of both pairs of $\lambda$-dependent eigenvalues in the complex plane is depicted in Figures 7, 8,9(a), and 9(b), from which it is evident that for all $\lambda<\lambda_{1}^{c}$, except for $\lambda<\lambda_{\text {cr }}$ (where a codimension-2 bifurcation occurs), the pairs of eigenvalues remain always in opposite planes of the Im axis, but symmetric with respect to the Re axis. This symmetry is always present for the pair with negative real parts, while for the other pair (with 


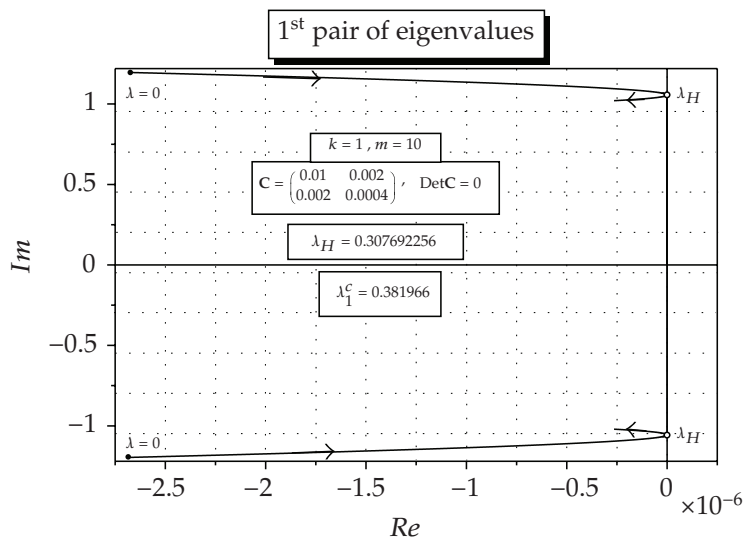

(a)

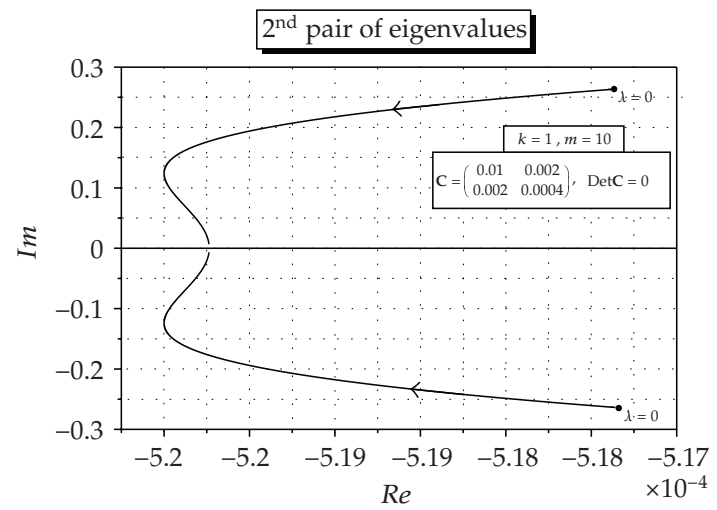

(b)

Figure 6: Evolution of both pairs of eigenvalues in the complex plane for the system of Case 3(a), associated with a degenerate Hopf bifurcation.

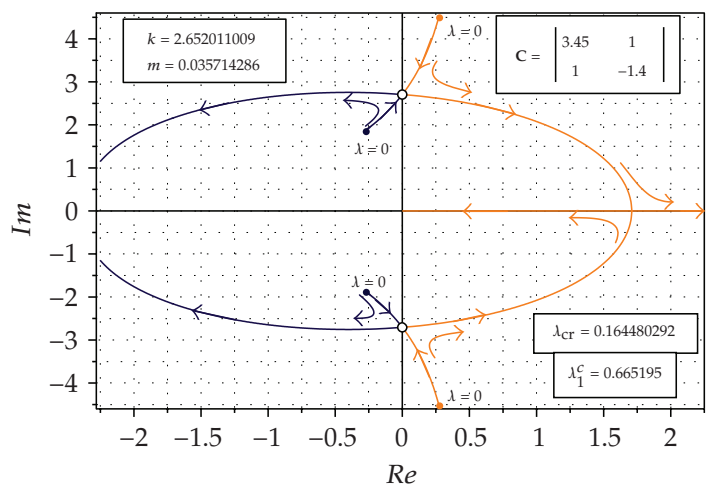

Figure 7: Evolution of both pairs of eigenvalues in the complex plane for the system of Case 3(d)1. 
Dimitris S. Sophianopoulos et al.

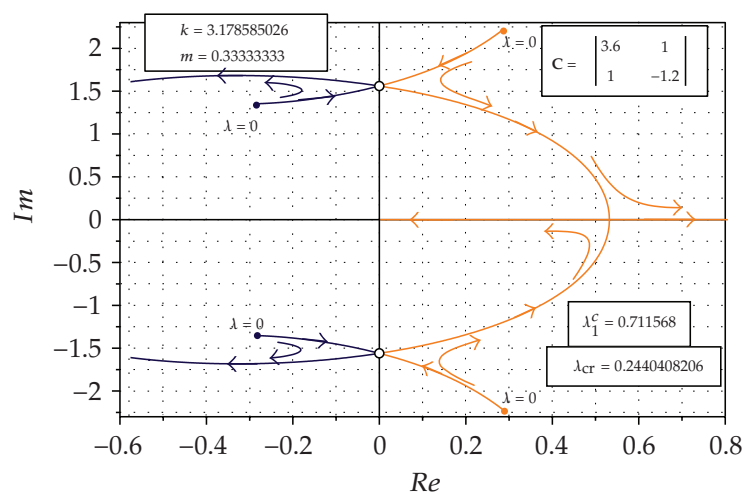

Figure 8: Evolution of both pairs of eigenvalues in the complex plane for the system of Case 3(d)2.

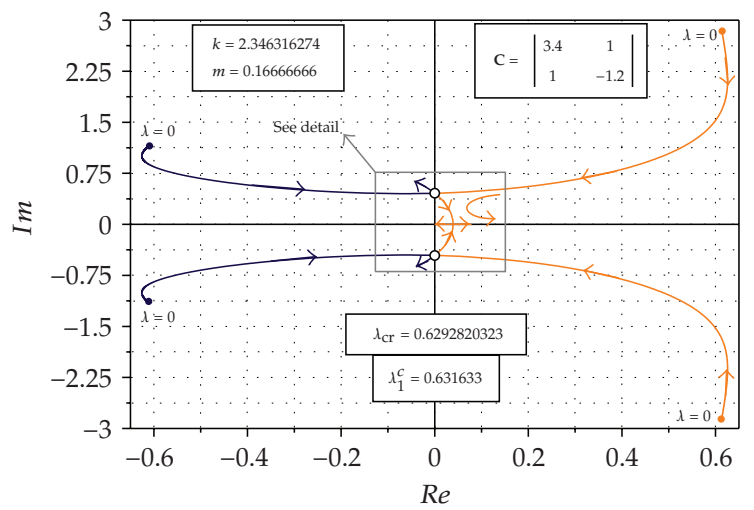

(a)

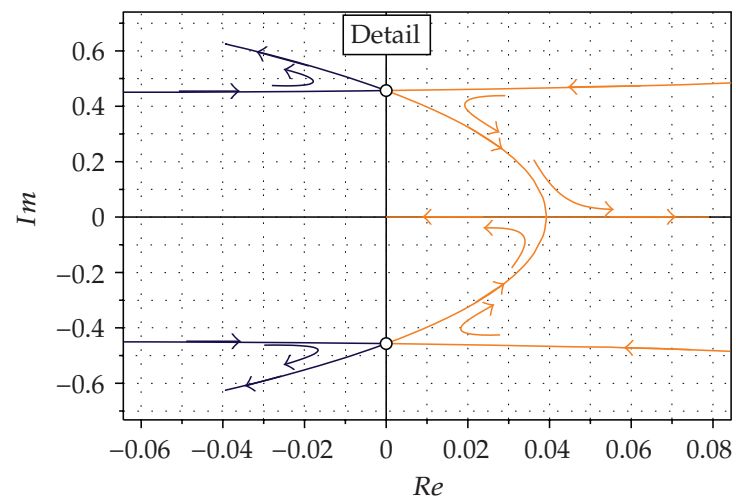

(b)

Figure 9: Evolution of both pairs of eigenvalues in the complex plane (a), and corresponding detail (b), for the system of Case 3(d)3. 


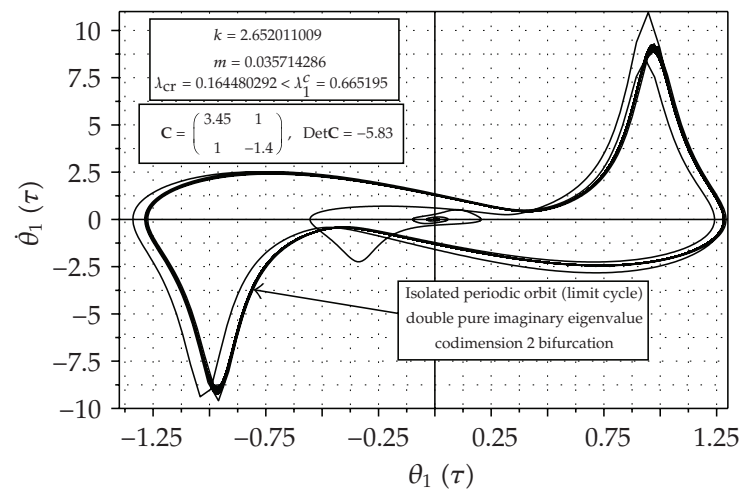

(a)

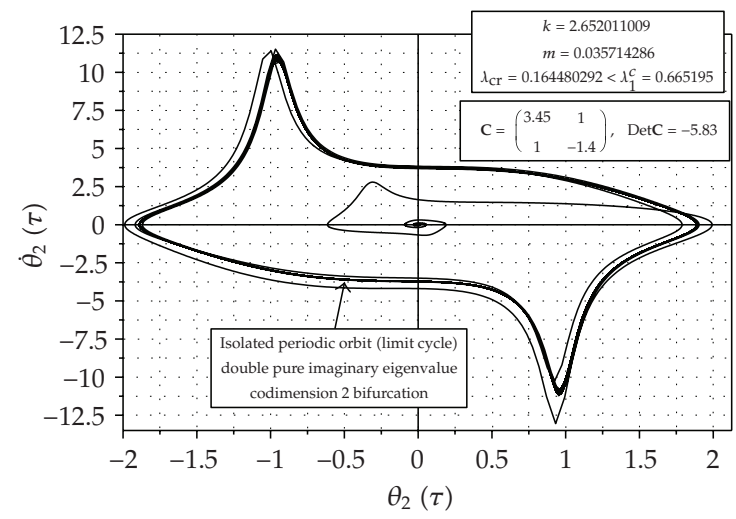

(b)

Figure 10: Phase-plane portraits $\left[\theta_{i}(\tau), \dot{\theta}_{i}(\tau)\right](i=1,2)$ for the system of Case $3(\mathrm{~d}) 1$, associated with an isolated periodic orbit.

positive real parts), this feature remains until their imaginary part vanishes simultaneously at a certain value of the loading $\lambda$ less than $\lambda_{1}^{c}$.

The dynamic response of the system for all these subcases is associated with isolated periodic orbits (whose final amplitude is constant and independent of the initial conditions), as shown in the phase-plane trajectories of Figures 10,11, and 12.

The corresponding dynamic bifurcations related to the above double pure imaginary eigenvalues behave like a generic Hopf bifurcation, whose basic feature is the intersection of the $\lambda$ dependent path of one eigenvalue with the imaginary axis. On the other hand, in all the above subcases, the branches of two consecutive $\lambda$-dependent eigenvalues meet the imaginary axis at the same point with $\lambda=\lambda_{\text {cr }}$. Namely, the transversality condition is satisfied through two intersected lines at the same point of the imaginary axis, but whose branches in the left (negative) and right (positive) half planes belong to the 1st and 2nd pairs of eigenvalues, respectively.

Finally, Figure 13 verifies the unexpected phenomenon (Kounadis [11]) of discontinuity in the dynamic loading $\lambda_{H}$ associated with either a degenerate or a generic Hopf bifurcation. 


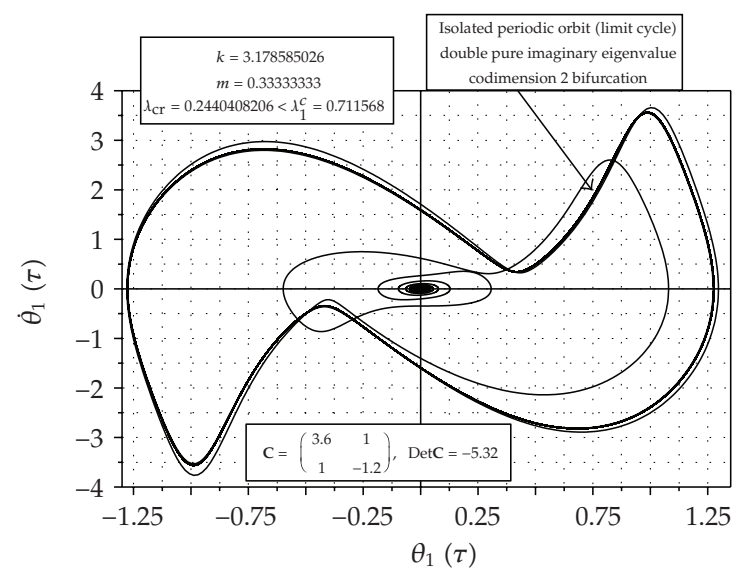

(a)

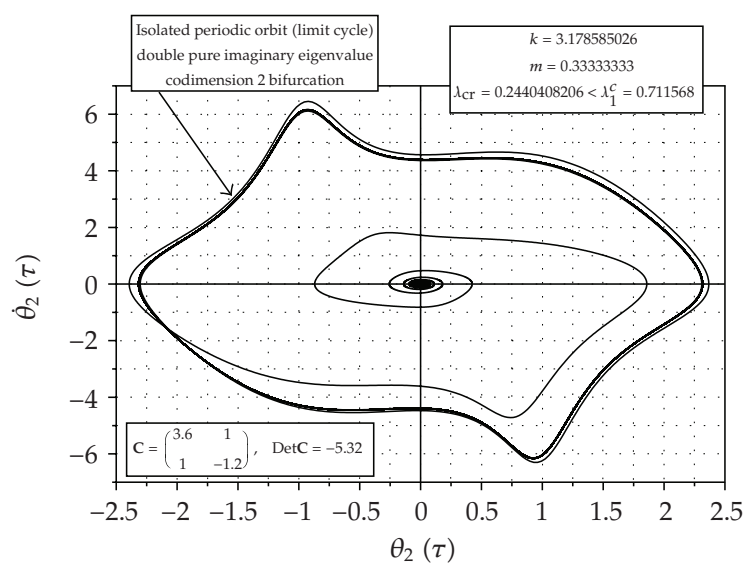

(b)

Figure 11: Phase-plane portraits $\left[\theta_{i}(\tau), \dot{\theta}_{i}(\tau)\right](i=1,2)$ for the system of Case $4 \mathrm{c} 2$, associated with an isolated periodic orbit.

\section{Concluding remarks}

This study discusses in detail the coupling effect of (infinitesimal mainly) damping with the mass and stiffness distribution in a 2-DOF cantilevered model under step potential loading. Such an autonomous system may be associated either with a positive semidefinite or indefinite damping matrix (with positive or negative diagonal elements). Attention is focused on the violation of the Lienard-Chipart stability criterion when this system is either unloaded or loaded by a suddenly applied load of constant magnitude and direction with infinite duration (step potential loading). The most important findings of this study are the following.

(1) Usage of Liénard-Chipart, simple and readily employed, stability criterion compared to that of Routh-Hurwitz brought into light a variety of new types of dynamic bifurcations reported below. 


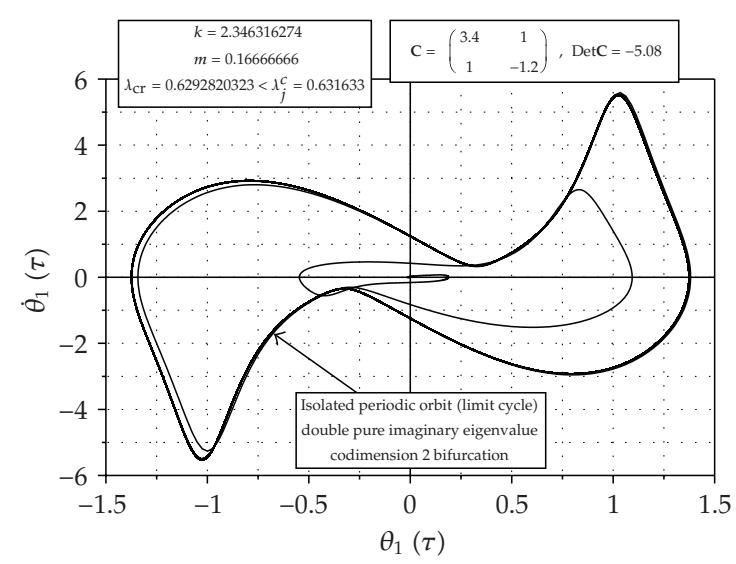

(a)

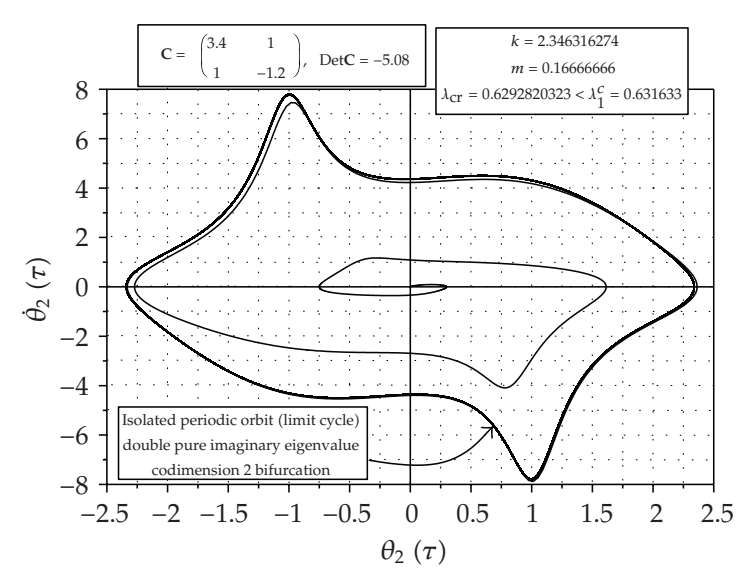

(b)

Figure 12: Phase-plane portraits $\left[\theta_{i}(\tau), \dot{\theta}_{i}(\tau)\right](i=1,2)$ for the system of Case 3(a), associated with an isolated periodic orbit.

(2) The mass and stiffness distribution combined either with a positive semidefinite or an indefinite damping matrix may have a considerable effect on the asymptotic stability, prior to divergence instability, even in case of infinitesimal damping.

(3) The cantilevered model when unloaded (being statically stable), strangely enough, under certain conditions becomes dynamically unstable to any small disturbance leading to a divergent (unbounded) motion.

(4) The above model when loaded under analogous of the previous conditions exhibits also a divergent motion at a certain value of the external load.

It is worth noting that both the above cases of divergent motion may occur for negligibly small negative determinant of the damping (indefinite) matrix when $\alpha_{1}=0$, while for $\alpha_{2}=0$ (regardless of whether

$\lambda=0$ or $\lambda \neq 0$ ), the determinant of the damping matrix is negative but finite. 


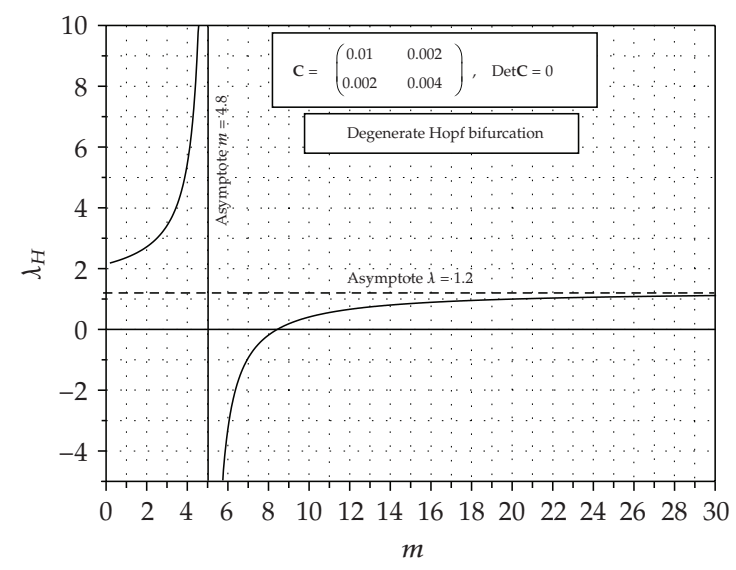

(a)

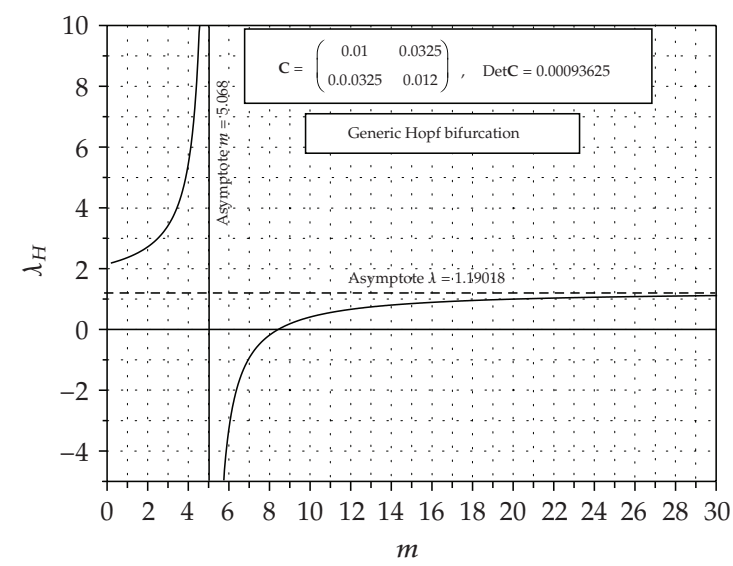

(b)

Figure 13: Plots $m$ versus $\lambda_{H}$ for a system with $k=1$ exhibiting a discontinuity for the case of (a) degenerate and (b) generic Hopf bifurcation.

(5) When $\Delta_{3}=0$ and $\alpha_{1} \alpha_{2} \neq 0$, four distinct responses may occur as follows.

(a) If $|c|=0$ (positive semidefinite damping matrix), the system exhibits a periodic motion associated with a degenerate Hopf bifurcation. Then, the final amplitude of motion depends on the initial conditions.

(b) When the damping matrix is indefinite (even with infinitesimal but negative determinant, $|c|<0$ ), the system may exhibit a periodic attractor response associated with a generic Hopf bifurcation. It is of paramount practical importance the case where such an unexpected dynamic instability occurs at a load $\lambda_{H}$ less than the 1st buckling load.

In both the above cases, it was confirmed the recently reported unexpected finding [11] of discontinuity of the dynamic loading $\lambda_{H}$ (associated with either a degenerate or a generic Hopf bifurcation) occurring at a certain value of the mass distribution. 
(c) When at the same time $\lambda=0$, we have a local dynamic instability for $|c| \leq 0$, whose global stability can be established through a nonlinear dynamic analysis.

(d) The case of a double pure imaginary eigenvalue may occur for an indefinite damping matrix with finite determinant and negative ratio of the corresponding diagonal elements. In this special case, there are two pairs of eigenvalues in the complex plane which touch the imaginary axis at the same point for a certain value $\lambda=\lambda_{\mathrm{cr}}$. This situation yields local instability leading to a motion with final constant amplitude regardless of the initial conditions. Namely, such a dynamic bifurcation behaves in a way similar to that of a generic Hopf bifurcation. This new type of dynamic bifurcation was also verified via a nonlinear dynamic analysis.

\section{Appendix}

\section{Results of symbolic computations}

(i) Conditions for $0<\lambda_{1}^{H}<\lambda_{1}^{c}$ from (3.20), with $c_{i i}>0(i=1,2), k>0, m>0$ are

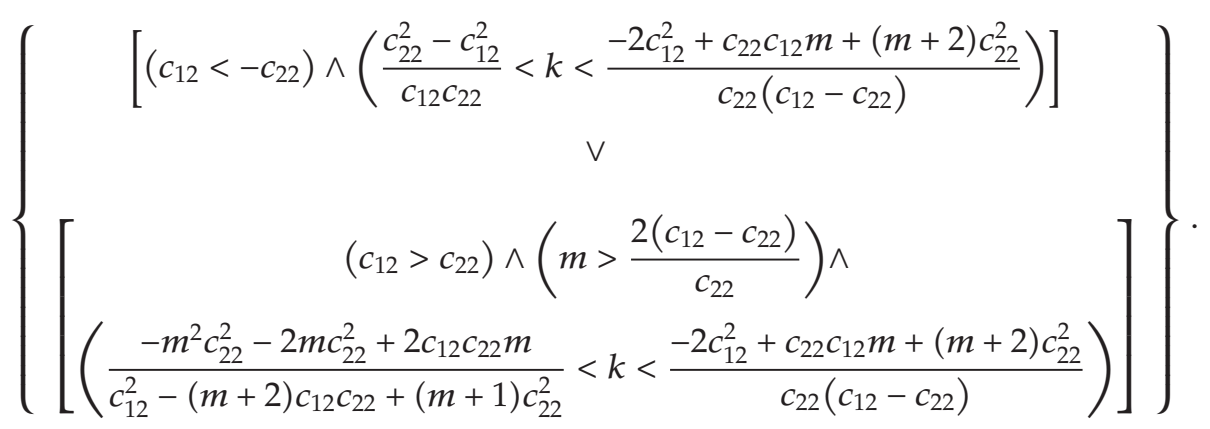

(ii) Expressions of coefficients $A_{i}(i=0,1,2)$ of $(3.23)$ are

$$
\begin{aligned}
& A_{2}=(k+m+2) c_{22}^{2}+2 c_{12}^{2}+4>0, \\
& A_{1}=\left\{\begin{array}{c}
c_{12}^{4}-2 c_{12} c_{22}\left(c_{22}^{2}-2\right)(k-m)+c_{12}^{2}\left[c_{22}^{2}(k+m-2)+8\right] \\
+c_{22}^{2}\left[(k-m)^{2}+c_{22}^{2}(k+1)(m+1)+4(k+m+2)\right]
\end{array}\right\}, \\
& A_{0}=\left[-2 c_{12}^{2}+c_{12} c_{22}(m-k)+c_{22}^{2}(k+m+2)\right]^{2} \geq 0,
\end{aligned}
$$

and corresponding values of $c_{11}, c_{22}$ for $|c|=0\left(\Leftrightarrow A_{0}=0\right)$ are

$$
\begin{gathered}
c_{22}=\frac{c_{12}}{k+m+2}\left[k-m+\sqrt{k^{2}-2 k(m-4)+(m+4)^{2}}\right]>0 \quad \forall c_{12} \neq 0, k>0, m>0, \\
c_{11}=\frac{2 c_{12}^{2}}{c_{22}}>0 \quad \forall c_{12} \neq 0, k>0, m>0 .
\end{gathered}
$$


Dimitris S. Sophianopoulos et al.

(iii) Values of $\lambda, k$, and $m$ for which the secular equation (3.7) has a double pure imaginary pair of roots (eigenvalues) are

$$
\begin{aligned}
k_{1,2} & =\frac{1}{2 c_{22}}\left(\begin{array}{c}
c_{22} c_{11}^{2}+\left(c_{22}^{2}-c_{12} c_{22} \mp \sqrt{c_{22}\left(c_{11}-2 c_{12}+c_{22}\right)\left(c_{11} c_{22}+4\right)}+2\right) c_{11} \\
-4 c_{12}+2 c_{22}-c_{22}\left(c_{12} c_{22}+\sqrt{c_{22}\left(c_{11}-2 c_{12}+c_{22}\right)\left(c_{11} c_{22}+4\right)}\right)
\end{array}\right), \\
\lambda_{1,2} & =\frac{1}{2}\left(c_{22}\left(c_{11}-c_{12}\right) \mp \sqrt{c_{22}\left(c_{11}-2 c_{12}+c_{22}\right)\left(c_{11} c_{22}+4\right)}+4\right), \\
m & =-\frac{c_{11}-2 c_{12}+c_{22}}{c_{22}}
\end{aligned}
$$

and the corresponding conditions for the above two sets of values to be positive are as follows.

For the 1 st set $\left(k_{1}, \lambda_{1}, m\right)$,

$$
\begin{gathered}
\left(-2<c_{22}<0\right) \wedge\left\{\begin{array}{c}
\left(-\sqrt{\frac{c_{11} c_{22}^{3}+4 c_{22}^{2}-4 c_{11} c_{22}-16}{c_{22}^{2}}}<c_{12}<\frac{c_{11}+c_{22}}{2}\right) \\
\left(-\frac{4}{c_{22}}<c_{11}<\frac{c_{22}^{2}}{8}\right) \wedge\left(\sqrt{\frac{c_{11} c_{22}^{3}+4 c_{22}^{2}-4 c_{11} c_{22}-16}{c_{22}^{2}}}<c_{12}<\frac{c_{11}+c_{22}}{2}\right)
\end{array}\right\} \\
\left(0<c_{22}<2\right) \wedge\left(\frac{c_{22}^{2}-8}{c_{22}}<c_{11} \leq-\frac{4}{c_{22}}\right) \wedge\left(\frac{c_{11}+c_{22}}{2}<c_{12}<-\sqrt{\frac{c_{11} c_{22}^{3}+4 c_{22}^{2}-4 c_{11} c_{22}-16}{c_{22}^{2}}}\right) .
\end{gathered}
$$

For the 2 nd set $\left(k_{2}, \lambda_{2}, m\right)$,

$$
\begin{array}{r}
\left(-2<c_{22}<0\right) \wedge\left[\begin{array}{c}
\left(-\frac{4}{c_{22}} \leq c_{11} \leq \frac{c_{22}^{2}-8}{c_{22}}\right) \wedge \mathbf{Q} \\
\vee \\
\left(c_{11}>\frac{c_{22}^{2}-8}{c_{22}}\right) \wedge \mathbf{X}
\end{array}\right] \\
\vee \\
\left(0<c_{22}<2\right) \wedge\left[\begin{array}{c}
\left(c_{11}<\frac{c_{22}^{2}-8}{c_{22}}\right) \wedge \mathbf{Y} \\
\vee \\
\left(\frac{c_{22}^{2}-8}{c_{22}} \leq c_{11}<-\frac{4}{c_{22}}\right) \wedge \mathbf{W} \\
\vee \\
\left(c_{11}=-\frac{4}{c_{22}}\right) \wedge \mathbf{Z}
\end{array}\right] .
\end{array}
$$


where

$$
\begin{aligned}
& \mathbf{Q}=\left(-\sqrt{\frac{c_{11} c_{22}^{3}+4 c_{22}^{2}-4 c_{11} c_{22}-16}{c_{22}^{2}}}<c_{12}<\frac{c_{11}+c_{22}}{2}\right) \\
& \mathbf{X}=\left(-\sqrt{\frac{c_{11} c_{22}^{3}+4 c_{22}^{2}-4 c_{11} c_{22}-16}{c_{22}^{2}}}<c_{12}<\sqrt{\frac{c_{11} c_{22}^{3}+4 c_{22}^{2}-4 c_{11} c_{22}-16}{c_{22}^{2}}}\right) \\
& \mathbf{Y}=\left(-\sqrt{\frac{c_{11} c_{22}^{3}+4 c_{22}^{2}-4 c_{11} c_{22}-16}{c_{22}^{2}}}<c_{12}<\sqrt{\frac{c_{11} c_{22}^{3}+4 c_{22}^{2}-4 c_{11} c_{22}-16}{c_{22}^{2}}}\right) \\
& \mathbf{W}=\left(\frac{c_{11}+c_{22}}{2}<c_{12}<\sqrt{\frac{c_{11} c_{22}^{3}+4 c_{22}^{2}-4 c_{11} c_{22}-16}{c_{22}^{2}}}\right), \\
& \mathbf{Z}=\left(\frac{c_{11}+c_{22}}{2}<c_{12}<-\sqrt{\frac{c_{11} c_{22}^{3}+4 c_{22}^{2}-4 c_{11} c_{22}-16}{c_{22}^{2}}}\right) .
\end{aligned}
$$

\section{References}

[1] H. Ziegler, “Die Stabilitätskriterien der Elastomechanik," Ingenieur Archiv, vol. 20, no. 1, pp. 49-56, 1952.

[2] A. N. Kounadis, "On the failure of static stability analyses of nonconservative systems in regions of divergence instability," International Journal of Solids and Structures, vol. 31, no. 15, pp. 2099-2120, 1994.

[3] A. N. Kounadis, "Non-potential dissipative systems exhibiting periodic attractors in regions of divergence," Chaos, Solitons E Fractals, vol. 8, no. 4, pp. 583-612, 1997.

[4] V. V. Bolotin, A. A. Grishko, and A. V. Petrovsky, "Secondary bifurcations and global instability of an aeroelastic non-linear system in the divergence domain," Journal of Sound and Vibration, vol. 191, no. 3, pp. 431-451, 1996.

[5] M. P. Païdoussis, D. Mateescu, and W.-G. Sim, "Dynamics and stability of a flexible cylinder in a narrow coaxial cylindrical duct subjected to annular flow," Journal of Applied Mechanics, vol. 57, no. 1, pp. 232-240, 1990.

[6] M. P. Païdoussis, Fluid-Structure Interactions: Slender Structures and Axial Flow, vol. 2, Elsevier Academic Press, London, UK, 2003.

[7] K. Huseyin, Multiple Parameter Stability Theory and Its Applications, vol. 18 of Oxford Engineering Science Series, Clarendon Press, Oxford, UK, 1986.

[8] F. R. Gantmacher, Lectures in Analytical Mechanics, Mir, Moscow, Russia, 1970.

[9] K. Huseyin, Vibrations and Stability of Damped Mechanical Systems, Sijthoff \& Noordhoff, Alphen aan den Rijn, The Netherlands, 1978.

[10] A. N. Kounadis, "Hamiltonian weakly damped autonomous systems exhibiting periodic attractors," Zeitschrift für Angewandte Mathematik und Physik, vol. 57, no. 2, pp. 324-350, 2006.

[11] A. N. Kounadis, "Flutter instability and other singularity phenomena in symmetric systems via combination of mass distribution and weak damping," International Journal of Non-Linear Mechanics, vol. 42, no. 1, pp. 24-35, 2007.

[12] E. E. Zajac, "The Kelvin-Tait-Chetaev theorem and extensions," Journal of the Astronautical Sciences, vol. 11, no. 2, pp. 46-49, 1964.

[13] E. E. Zajac, "Comments on "stability of damped mechanical systems and a further extension"," AIAA Journal, vol. 3, no. 9, pp. 1749-1750, 1965.

[14] R. Sygulski, "Dynamic stability of pneumatic structures in wind: theory and experiment," Journal of Fluids and Structures, vol. 10, no. 8, pp. 945-963, 1996. 
[15] A. Laneville and A. Mazouzi, "Wind-induced ovalling oscillations of cylindrical shells: critical onset velocity and mode prediction," Journal of Fluids and Structures, vol. 10, no. 7, pp. 691-704, 1996.

[16] A. K. Misra, S. S. T. Wong, and M. P. Païdoussis, "Dynamics and stability of pinned-clamped and clamped-pinned cylindrical shells conveying fluid," Journal of Fluids and Structures, vol. 15, no. 8, pp. 1153-1166, 2001.

[17] A. N. Kounadis, "A geometric approach for establishing dynamic buckling loads of autonomous potential two-degree-of-freedom systems," Journal of Applied Mechanics, vol. 66, no. 1, pp. 55-61, 1999.

[18] F. R. Gantmacher, The Theory of Matrices, Chelsea, New York, NY, USA, 1959.

[19] L. A. Pipes and L. R. Harvill, Applied Mathematics for Engineers and Physicists, International Student Edition, McGraw-Hill, Kogakusha, Tokyo, Japan, 3rd edition, 1970.

[20] A. T. Fuller, "Conditions for a matrix to have only characteristic roots with negative real parts," Journal of Mathematical Analysis and Applications, vol. 23, no. 1, pp. 71-98, 1968.

[21] T. B. Bahder, Mathematica for Scientists and Engineers, Addison-Wesley, Reading, Mass, USA, 1995. 


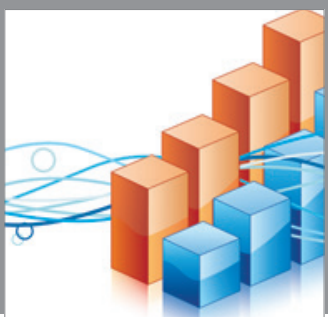

Advances in

Operations Research

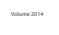

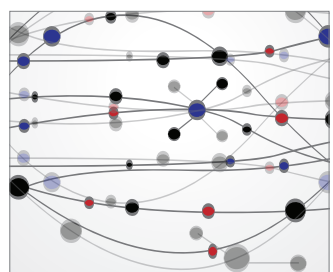

\section{The Scientific} World Journal
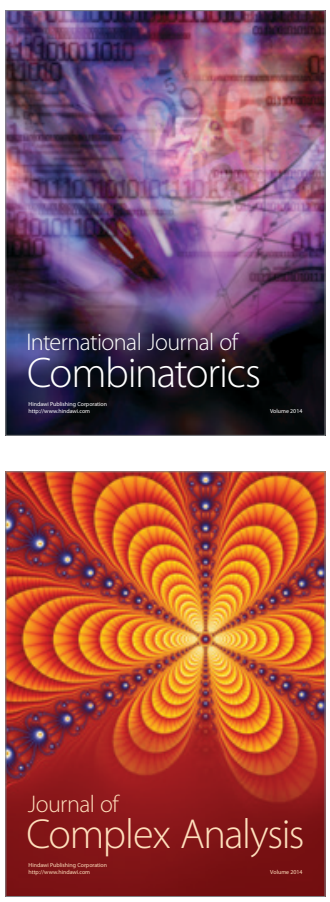

International Journal of

Mathematics and

Mathematical

Sciences
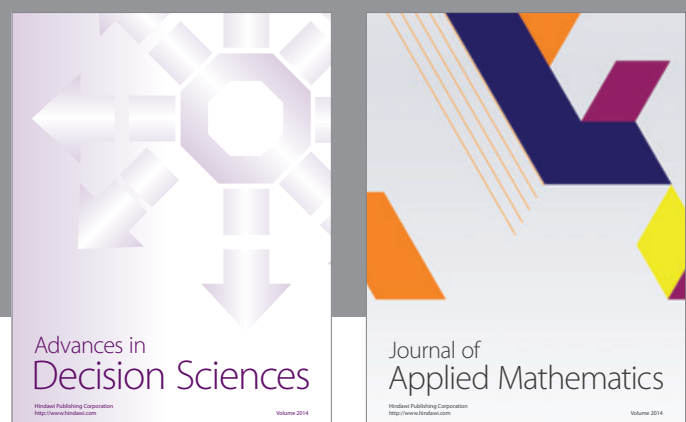

Journal of

Applied Mathematics
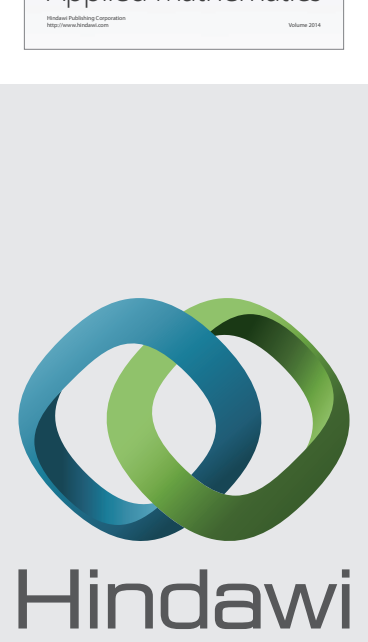

Submit your manuscripts at http://www.hindawi.com
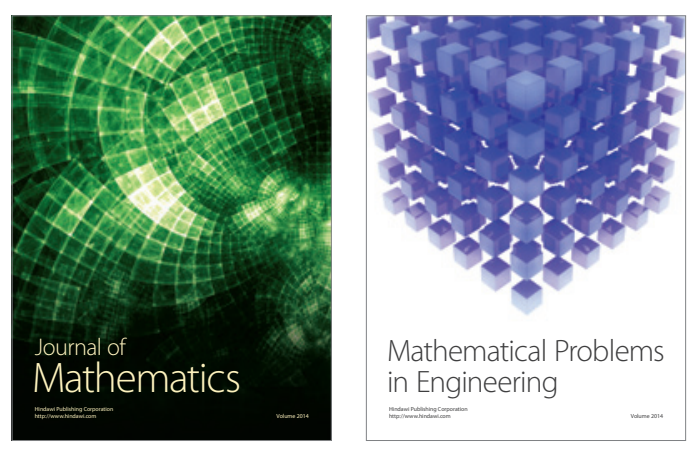

Mathematical Problems in Engineering
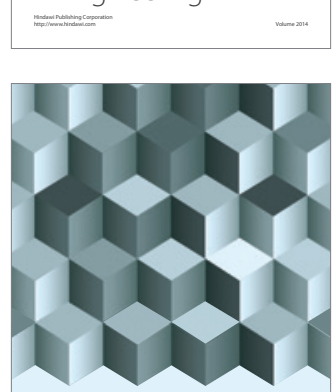

Journal of

Function Spaces
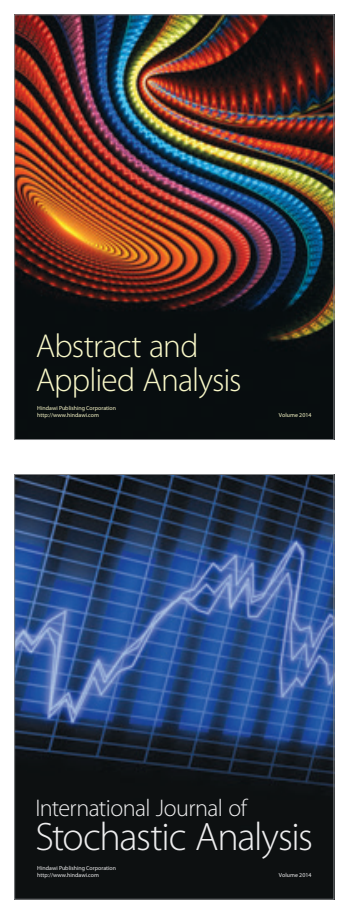

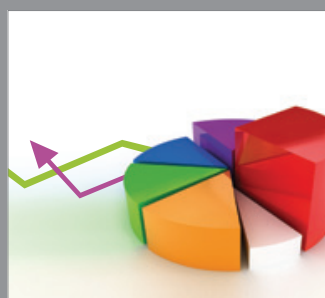

ournal of

Probability and Statistics

Promensencen
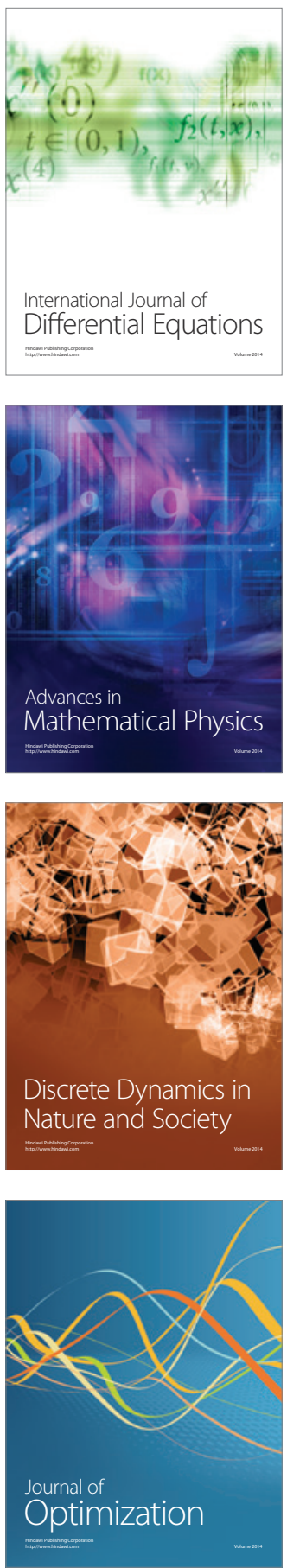